\title{
Gefahren des Piercing: Welcher Erreger hat hier zugeschlagen?
}

— ine 18-jährige Frau suchte wegen einer ausgedehnten, — rötlich livide verfärbten und fluktuierenden Hautveränderung im Bereich der rechten Wange eine Hautklinik auf (Abb. links). Vor sechs Monaten hatte sie sich in einem einschlägigen Etablissement ein Piercing am rechten Tragus anbringen lassen. Etwa vier Wochen später war die strangförmige Hautveränderung aufgetreten. Der Hausarzt hatte bereits einen Abszess in dieser Gegend drainiert und mit Cefalexin behandelt, was den Zustand aber nicht verbesserte. Um eine Infektion mit atypischen Erregern auszuschließen, führte man eine Biopsie durch. Dabei wurde eine granulomatöse Entzündung festgestellt, es fanden sich aber keine säurefesten Stäbchen. Kulturell konnte Mycobacterium fortuitum nachgewiesen werden. Nach einer vier Monate langen Behandlung mit Ciprofloxacin und Trimethoprim-Sulfamethoxazol trat eine deutliche klinische Besserung ein (Abb. rechts). Die Verordnung eines Makrolids wurde wegen der gleichzeitigen Einnahme von Betablockern vermieden.

Fazit: Für den Kunden von Piercing-Studios ist es nicht einfach herauszufinden, ob dort einschlägige hygienische Standards eingehalten werden. Abgesehen von den methodenimmanenten Problemen wie Perichondritis, unspezifischen Entzündungen oder Abszedierungen können Infektionen mit Hepatitis B oder C auftreten. Heilt ein entzündlicher Lokalbefund unter Lokaltherapie und systemischer antibiotischer Behandlung nicht ab, sollte an die Infektion mit einem atypischen Erreger gedacht werden. Säurefeste Stäbchen sind nicht immer im histologischen Präparat nachweisbar, sodass man eine Isolierung des Erregers in der Gewebekultur versuchen sollte. Nicht tuberkulöse Mykobakterien verursachen meist Pusteln, Plaques und Ulzerationen mit ausgesprochen schlechter Abheilungstendenz.

H. S. FüeßI

Horii KA, Jackson MA. Piercing-related nontuberculous mycobacterial infection. New Engl J Med 2010; 362: 21.
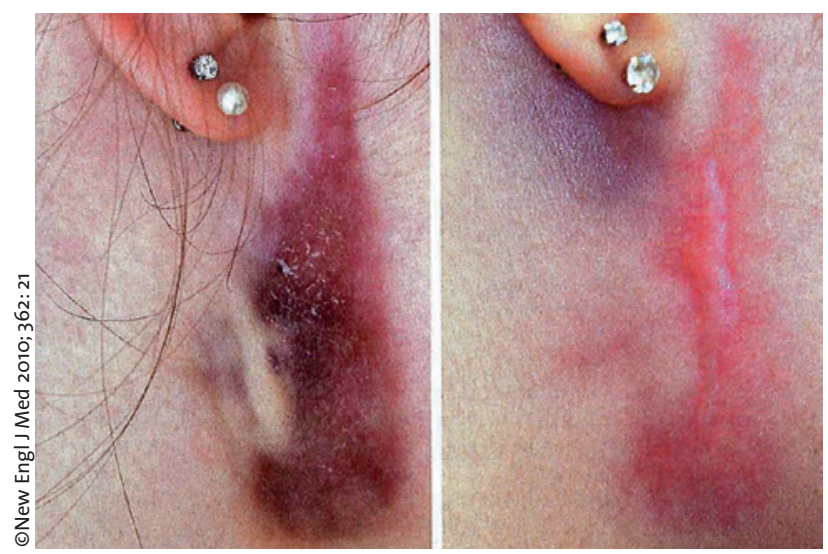

Hautveränderung vor (links) und nach viermonatiger antibiotischer Therapie (rechts). 